\title{
What is the efficacy of switching to weekly pegvisomant in acromegaly patients well controlled on combination therapy?
}

\author{
A Muhammad', A J van der Lely ', R D O'Connor', P J Delhanty', J Dal', \\ A H Dallenga ${ }^{1}$, R A Feelders ${ }^{1}$, J A M J L Janssen ${ }^{1}$, J O L Jorgensen ${ }^{2}$ and \\ S J C M M Neggers' \\ 'Endocrinology Section, Department of Medicine, Pituitary Center Rotterdam, Erasmus University \\ Medical Center, Rotterdam, The Netherlands and ${ }^{2}$ Medical Department (Endocrinology and Diabetes), \\ Medical Research Laboratories, Clinical Institute, Aarhus, Denmark
}

\author{
Correspondence \\ should be addressed \\ to A Muhammad \\ Email \\ a.muhammad.1@ \\ erasmusmc.nl
}

\begin{abstract}
Context: Although combination therapy of acromegaly with long-acting somatostatin analogs (LA-SSAs) and pegvisomant (PEGV) normalizes insulin-like growth factor-1 (IGF1) levels in the majority of patients, it requires long-term adherence. Switching from combination therapy to monotherapy with weekly PEGV could improve patients' comfort, but the efficacy is unknown.

Objective: To assess the efficacy of switching to PEGV monotherapy in patients well controlled on combination therapy of LA-SSAs and PEGV.

Design: Single-center, open-label observational pilot study. LA-SSA therapy was discontinued at baseline and all patients were switched to PEGV monotherapy for 12 months. If IGF1 levels exceeded 1.0 times upper limit of normal (ULN), PEGV dose was increased by $20 \mathrm{mg}$ weekly.

Subjects and methods: The study included 15 subjects (eight males), with a median age of 58 years (range 35-80) on combination therapy of high-dose LA-SSAs and weekly PEGV for $>6$ months, and IGF1 levels within the normal range. Treatment efficacy was assessed by measuring serum IGF1 levels.

Results: After 12 months of weekly PEGV monotherapy, serum IGF1 levels of $73 \%$ of the subjects remained controlled. In one patient, LA-SSA had to be restarted due to recurrence of headache. IGF1 levels increased from a baseline level of $0.62 \times$ ULN (range $0.30-0.84)$ to $0.83 \times$ ULN $(0.30-1.75)$ after 12 months, while the median weekly PEGV dose increased from $60(30-80) \mathrm{mg}$ to $80(50-120) \mathrm{mg}$.

Conclusion: Our results suggest that switching from combination therapy of LA-SSAs and PEGV to PEGV monotherapy can be a viable treatment option for acromegaly patients without compromising efficacy.

\section{Introduction}

Acromegaly is a rare disease characterized by somatic overgrowth and endocrine dysfunction due to excessive secretion of growth hormone $(\mathrm{GH})$ and subsequent elevation of insulin-like growth factor-1 (IGF1) levels. In more than $90 \%$ of patients, it is caused by a benign growth hormone-secreting pituitary adenoma (1). In acromegaly patients, the main causes of death are due to cardiovascular
두 2016 European Society of Endocrinology Printed in Great Britain and respiratory diseases $(2,3)$. Normalization of $\mathrm{GH}$ and IGF1 levels will result in normal mortality rates, reduced morbidity, and a reduction in symptoms (3).

The GH receptor antagonist pegvisomant (PEGV) is the medical treatment that has the highest reported efficacy (4). PEGV can be administered with or without long-acting somatostatin analogs (LA-SSAs) $(5,6)$. 
Administration of PEGV alone at a mean weekly dose of $130 \mathrm{mg}$ can normalize IGF1 levels in over $90 \%$ of patients (7). If LA-SSAs and PEGV are used together, a similar efficacy of over $90 \%$ is achieved. However, a considerably lower mean weekly dose of 77-80 mg PEGV is required; this may result in a more cost-effective treatment.

Both LA-SSAs and PEGV are administered, respectively, as i.m. and s.c. injections. LA-SSAs are injected every 4 weeks, while PEGV can be injected either as a daily or as a weekly injection. While most studies on efficacy have been based on clinical trials that evaluated the daily injection $(7,8)$, only one study has assessed the weekly injection. Higham et al. (9) reported a normalization of IGF1 in $71 \%$ of acromegaly patients $(n=7)$ treated with weekly PEGV monotherapy.

Switching from combined therapy of LA-SSAs and PEGV to PEGV monotherapy in pateints can reduce the total number of injections, and therefore improve patient adherence. In addition, PEGV monotherapy allows for easier dose adaptation.

In this pilot study, we assessed the effect of switching from combination therapy to weekly PEGV monotherapy. The main outcome parameters were the proportion of patients with normalized IGF1 levels after 12 months follow-up with weekly PEGV monotherapy.

\section{Subjects and methods}

\section{Patients}

In 2009, a prospective observational study enrolled 15 subjects (8 males) with acromegaly from a single center, who were treated with a combination of LA-SSAs (13 lanreotide autogel, 2 octreotide LAR) and PEGV (Table 1). These patients received a combination therapy because their serum IGF1 levels exceeded the normal range or because their acromegaly symptoms persisted during high dose of LA-SSA monotherapy (5). Inclusion criteria for this study were a stable LA-SSA dose, the use of PEGV as a weekly injection, and biochemical remission for over 6 months before enrolment, defined by an IGF1 within the normal range for sex and age of $<1.0$ upper limit of normal (ULN) (8). The cause of acromegaly needed to be a GH-secreting pituitary adenoma and the allowed dose of weekly PEGV was $\leq 80 \mathrm{mg}$. The median age of the study group was 58 (range 35-80) years. Three patients had undergone surgery of the pituitary adenoma in the past, while six patients had undergone with both surgery and radiotherapy. Radiotherapy was administered at least 5 years before study entry. Six patients had only received
Table 1 Baseline characteristics of all subjects $(n=15)$.

\begin{tabular}{|c|c|}
\hline Characteristics & $\boldsymbol{n}(\%)$ \\
\hline \multicolumn{2}{|l|}{ Demographics } \\
\hline Sex, female & $8(53.3)$ \\
\hline Age, median (range) & $58(35-80)$ \\
\hline Diabetes mellitus type II & $3(20)$ \\
\hline \multicolumn{2}{|l|}{ Previous treatments } \\
\hline Transsphenoidal surgery & $3(20)$ \\
\hline $\begin{array}{l}\text { Transsphenoidal surgery } \\
\text { and radiotherapy }\end{array}$ & $6(40)$ \\
\hline Primary medical therapy & $6(40)$ \\
\hline $\begin{array}{l}\text { Weekly PEGV dose } \\
\text { (mg; median (range)) }\end{array}$ & $60(9.3-33.4)$ \\
\hline \multicolumn{2}{|c|}{ Serum assessments, median (range) } \\
\hline IGF1 ( $\mathrm{nmol} / \mathrm{L})$ & $15.7(9.3-33.4)$ \\
\hline IGF1 $\times$ ULN & 0.62 of ULN $(0.30-0.84)$ \\
\hline $\mathrm{GH}(\mu \mathrm{g} / \mathrm{L})$ & $3.03(0.19-15.95)$ \\
\hline Glucose (mmol/L) & $5.4(3.4-20)$ \\
\hline $\mathrm{HbA1c}(\mathrm{mmol} / \mathrm{L})$ & $6.1(5.1-9.2)$ \\
\hline \multicolumn{2}{|c|}{ Long-acting somatostatin analogs } \\
\hline Lanreotide autogel & $13(87)$ \\
\hline Octreotide LAR & $2(13)$ \\
\hline
\end{tabular}

medical treatment. Three patients had diabetes and were on oral medication, while one diabetes patient also used insulin treatment. All patients gave their written informed consent, and the study was approved by our local institutional review board.

One patient had to be restarted with LA-SSAs during the study period due to a recurrence of symptoms and therefore did not want to continue with the PEGV alone. This patient was reported as uncontrolled and was censored from the results after withdrawal, but was counted as treatment failure.

\section{Study}

At the start of the study, LA-SSAs were discontinued for 12 months and only PEGV was continued. Every 6 weeks, patients visited our out-patient clinic to measure IGF1, hemoglobin A1c (HbA1c), glucose, insulin, cholesterol, free fatty acids, and triglycerides levels. During the visits, data on symptoms and safety assessments were collected and the dose of PEGV was adjusted if necessary. IGF1 levels were measured by immunometric assays (Diagnostic Products Corp., Los Angeles, CA, USA) and were interpreted according to the sex-dependent and agedependent ranges (10). GH and serum PEGV levels were measured at the start of the trial, after 6 months, and after 12 months. As endogenous GH was in the presence of PEGV, GH levels were measured using a specific assay free of interference of the drug (11). When IGF1 levels exceeded the normal range, the PEGV dose was further increased by $20 \mathrm{mg} /$ week. This was continued until the 
IGF1 levels were within the normal range for sex and age and PEGV dose was not changed. Doses were divided into two equal portions per week when PEGV exceeded $80 \mathrm{mg}$. Efficacy of PEGV monotherapy was assessed 12 months after discontinuation of the combination therapy. Safety assessment included measurement of serum alkaline phosphatase, $\gamma$-glutamyltranspeptidase, alanine aminotransferase, aspartate aminotransaminase, lactate dehydrogenase, and total bilirubin. A pituitary MRI was performed before study entry and after the study.

\section{Statistical analysis}

Prism version 5.00 for windows (GraphPad Software) was used for the statistical analyses of the data. Although the nature of the study was mainly descriptive, pertinent data were analyzed using Wilcoxon's signed-rank test. Statistical significance was defined by $P<0.05$ (two-tailed). Data were expressed as median \pm (range), unless specified otherwise.

\section{Results}

\section{Efficacy of combination therapy vs PEGV monotherapy}

Fifteen patients were enrolled in this study. At baseline, the IGF1 levels of all patients were controlled with a median IGF1 level of $62 \%$ of the ULN (range 30-84\%) (Fig. 1). At 6 months, IGF1 levels started to increase to

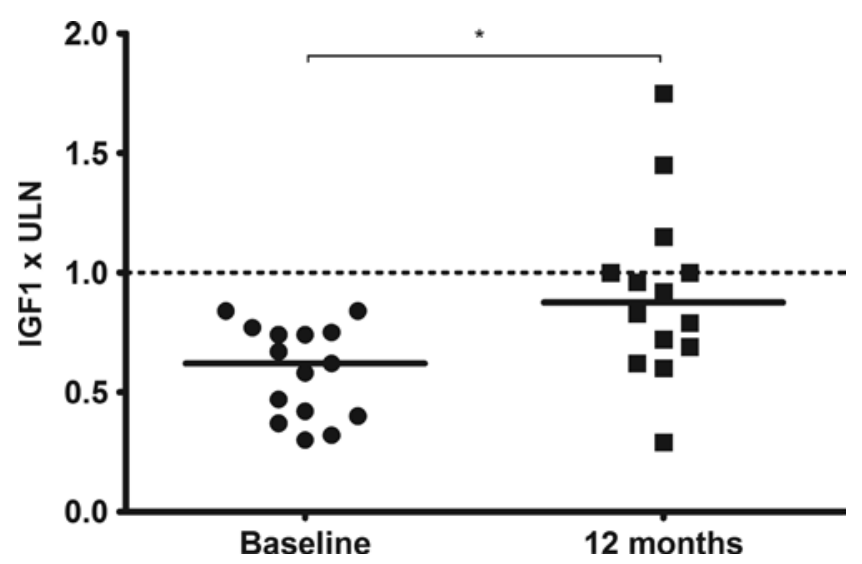

Figure 1

IGF1 levels at baseline and after 12 months of pegvisomant therapy. IGF1 levels expressed as times upper limit of normal, at baseline $(n=15)$ and after 12 months $(n=14)$. One patient restarted somatostatin analogs due to recurrence of symptoms.
$100 \%$ of the ULN (range $43-220 \%$ ). The slow increase of IGF1 started 12 weeks after cessation of the LA-SSA. At baseline, patients were using PEGV at a median dose of $60 \mathrm{mg}$ (range 30-80 mg). After 12 months, median IGF1 levels increased to $83 \%(30-175 \%)$ of the ULN $(P<0.05$; Fig. 1). The median PEGV dose had been increased to $80 \mathrm{mg}$ (50-120 mg; $P<0.05)$. IGF1 remained controlled in 11 of 15 subjects (73\%) after 12 months of weekly PEGV monotherapy.

At the start of the study, median serum PEGV levels were $3.00 \mathrm{mg} / \mathrm{L}(0.25-19.44)$. After an initial decrease in serum PEGV levels $2.23(0.44-12.10)$ at 6 months, PEGV levels increased to $4.36 \mathrm{mg} / \mathrm{L}$ (range 1.12-21.28) at 12 months. Between 6 and 12 months, the serum PEGV levels were significantly higher $(P=0.01)$, but no difference was found between baseline and 6 or 12 months.

We compared PEGV levels between the subjects in whom IGF1 levels were elevated and those who had levels that remained within the normal range after 12 months. We observed that after 12 months, acromegaly patients with an elevated IGF1 tended to have lower PEGV levels, this was $3.85 \mathrm{mg} / \mathrm{L}(1.30-4.39 \mathrm{mg} / \mathrm{L})$ in noncontrolled patients compared with $5.54 \mathrm{mg} / \mathrm{L}(1.12-21.28 \mathrm{mg} / \mathrm{L})$ in controlled patients $(P=0.25)$. Weekly PEGV doses and increases in doses were not different between controlled and noncontrolled patients: the baseline median PEGV dose was $60(30-80 \mathrm{mg})$ in controlled vs $60(40-80 \mathrm{mg})$ in noncontrolled patients. After 12 months, the respective median PEGV dose increased to $80(50-120 \mathrm{mg}$ ) in controlled vs 100 (80-120 mg) in noncontrolled patients. The median increase in PEGV dose was $+10(0-60 \mathrm{mg})$ in those who were controlled after 12 months, while this was $+40(20-40 \mathrm{mg})$ for those who were noncontrolled.

Five subjects did not require changes in PEGV dosing, because their IGF1 level remained within the age-adjusted normal limits. All five subjects had received combination therapy longer period (5.88 (2.26-6.01) years) than the other 10 subjects (2.52 (1.48-6.39) years).

Over time, the GH levels tended to increase during PEGV monotherapy. At baseline, we measured 2.99 $\mu \mathrm{g} / \mathrm{L}$ (range $0.19-15.95 \mu \mathrm{g} / \mathrm{L}$ ) compared with $3.22 \mu \mathrm{g} / \mathrm{L}$ $(0.12-79.14 \mu \mathrm{g} / \mathrm{L}$ ) at 6 months, and $6.36 \mu \mathrm{g} / \mathrm{L}$ (range $0.180-31.45 \mu \mathrm{g} / \mathrm{L}$ ) at 12 months. The GH levels at baseline and 6 and 12 months were significantly different $(P<0.05)$, except for baseline vs 12 months $(P=0.055)$.

\section{Safety assessment}

No significant safety issues were observed during PEGV monotherapy. After 12 months, glycosylated HbA1c 
decreased significantly from $6.0 \%(5.1-9.2 \%)$ to $5.9 \%$ (5.0-9.1\%; $P=0.02)$. The patients with diabetes had no alterations to their anti-diabetic medication during the study.No significant elevation of liver enzymes was observed and the MRI of pituitary did not show any change in tumor size. One patient who withdrew from the study had severe headaches, which was resolved after reinitiating LA-SSAs.

\section{Discussion}

In this study, we followed 15 acromegaly patients who were previously biochemically controlled with LA-SSAs and weekly PEGV. These patients had been switched to combination therapy because their IGF1 levels could not be controlled by LA-SSA monotherapy. In this study, we discontinued LA-SSAs and continued with weekly PEGV monotherapy. At baseline, $100 \%$ of the subjects had IGF1 levels within the normal limits during combination therapy. After 12 months follow-up, 73\% remained controlled.

The efficacy of $73 \%$ is in the same range as the efficacy of $68 \%$ reported by the ACROSTUDY group. However, we achieved this proportion of IGF1 normalization at a median PEGV dose of $80 \mathrm{mg} /$ week after 12 months, whereas the ACROSTUDY group reported this at a median PEGV dose of $17.2 \mathrm{mg} /$ day after 5 years, which corresponds to approximately $120 \mathrm{mg} /$ week (12). The subjects enrolled in our study used relatively low doses of PEGV and had used the combination with LA-SSAs for at least 1.5 years. As this may have had an impact on our results, these results cannot be extrapolated to all patients using combination therapy.

The slow and gradual increase in serum IGF1 levels that we observed 16 weeks after discontinuation of LA-SSAs is in line with the expected washout time of the LA-SSAs and the carryover effect of the LA-SSAs of about 15-20 weeks $(5,13,14)$. During 12-month follow-up, 5 of 15 patients did not need any dose adaptation. Although IGF1 levels also increased in these patients, they remained within the age-adjusted normal range. Combination therapy in these five patients lasted for longer period, than for the other patients in the study. A longer period of treatment with LA-SSA might result in prolonged 'suppression' of GH, which may improve the efficacy of PEGV, a competitive inhibitor of the $\mathrm{GH}$ receptor (4). In the total population, single measurements of $\mathrm{GH}$ tended to increase at 6 and 12 months during the study. However, subjects in whom IGF1 remained within the normal range tended to have lower GH levels than those with elevated IGF1 levels.
Subjects in whom IGF1 levels increased after the discontinuation of the LA-SSAs tended to have lower PEGV serum levels, despite treatment with similar doses of PEGV. One would expect that PEGV levels would decrease after discontinuation of LA-SSAs, because LASSAs increase the PEGV serum levels by approximately $20 \%$ compared with an identical dose of PEGV alone $(6,11)$. This observation explains the difference in PEGV levels between controlled and uncontrolled patients at 6 months, but not the difference in PEGV levels at 12 months. A potential explanation is that uncontrolled patients do not increase in PEGV serum levels as much as controlled patients when the weekly dose of PEGV is increased. The large difference in serum PEGV levels between subjects has been reported previously (15). Possibly due to the small sample size of our study, however, we found no association between PEGV dose and previously reported characteristics such as adiposity and body weight.

One patient who was discussed in the safety analysis was withdrawn from the study because she suffered from severe headaches, even though serum IGF1 level was controlled (98\% of ULN). After recommencing LASSA cotreatment, the headache disappeared. The pituitary MRI scan showed no explanation for the headache. In this specific case, we suspect that there was a causal relationship between discontinuation of LA-SSA and headache. As expected, HbA1c levels decreased significantly in these patients in our study. However, the change was very small, and therefore of dubious clinical relevance.

This was a pilot study on monotherapy with weekly PEGV to assess whether ceasing LA-SSAs is a viable option in a selection of our patients on combination therapy. A drawback of this pilot study is the small sample size. Nevertheless, our results suggest that a significant number of acromegaly patients on low-dose PEGV in combination with LA-SSAs can temporarily stop their LA-SSA treatment for at least a year. These treatment interruptions may improve patient adherence to the medication and reduce the economic burden of long-term use of expensive medications.

We conclude that discontinuing LA-SSAs and continuing with PEGV may be an interesting alternative for a selected group of patients that are well controlled with a combination of LA-SSAs and relatively low doses of PEGV. It is possible to maintain biochemical control up to 12 months in a substantial number of patients without raising the dose of PEGV. In patients who have previously been treated for an extended time with LA-SSAs, there is no need for adjust therapy even after 12 months. It seems 
sensible to determine the usability of structured interruptions in medical therapy in patients with acromegaly in a larger study.

\section{Declaration of interest}

A J van der Lely is consultant for Novartis Pharma, Pfizer International and received grants from Novartis Pharma, Ipsen Pharma International, and Pfizer International. S N received research grant from Ipsen and Pfizer. $\mathrm{J} \mathrm{O} \mathrm{L} \mathrm{J}$ is a consultant for Pfizer and Novartis and has received lecture fees and unrestricted research grants from Novartis, Pfizer, and IPSEN. The other authors have nothing to disclose.

\section{Funding}

This research did not receive any specific grant from any funding agency in the public, commercial, or not-for-profit sector.

\section{References}

1 Melmed S. Medical progress: acromegaly. New England Journal of Medicine 2006355 2558-2573. (doi:10.1056/NEJMra062453)

2 Colao A, Ferone D, Marzullo P \& Lombardi G. Systemic complications of acromegaly: epidemiology, pathogenesis, and management. Endocrine Reviews 200425 102-152. (doi:10.1210/JC.2015-1589)

3 Holdaway IM, Bolland MJ \& Gamble GD. A meta-analysis of the effect of lowering serum levels of GH and IGF-I on mortality in acromegaly. European Journal of Endocrinology 2008159 89-95. (doi:10.1111/ cen.2011.75.issue-4)

4 Neggers SJ, Franck SE, de Rooij FW, Dallenga AH, Poublon RM, Feelders RA, Janssen JA, Buchfelder M, Hofland LJ, Jorgensen JO et al. Long-term efficacy and safety of pegvisomant in combination with long-acting somatostatin analogues in acromegaly. The Journal of Clinical Endocrinology and Metabolism 201499 3644-3652. (doi:10.1210/jc.2014-2032)

5 Chen T, Miller TF, Prasad P, Lee J, Krauss J, Miscik K, Kalafsky G \& McLeod JF. Pharmacokinetics, pharmacodynamics, and safety of microencapsulated octreotide acetate in healthy subjects. Journal of Clinical Pharmacology 200040 475-481. (doi:10.1177/00912700022009242)

6 van der Lely AJ, Muller A, Janssen JA, Davis RJ, Zib KA, Scarlett JA $\&$ Lamberts SW. Control of tumor size and disease activity during cotreatment with octreotide and the growth hormone receptor antagonist pegvisomant in an acromegalic patient. Journal of Clinical Endocrinology and Metabolism 200186 478-481. (doi:10.1210/jcem.86.2.7206)

7 van der Lely AJ, Hutson RK, Trainer PJ, Besser GM, Barkan AL, Katznelson L, Klibanski A, Herman-Bonert V, Melmed S, Vance ML et al. Long-term treatment of acromegaly with pegvisomant, a growth hormone receptor antagonist. Lancet 2001358 1754-1759. (doi:10.1016/S0140-6736(01)06844-1)

8 Trainer PJ, Drake WM, Katznelson L, Freda PU, Herman-Bonert V, van der Lely AJ, Dimaraki EV, Stewart PM, Friend KE, Vance ML et al. Treatment of acromegaly with the growth hormone-receptor antagonist pegvisomant. New England Journal of Medicine $2000 \mathbf{3 4 2}$ 1171-1177. (doi:10.1056/NEJM200004203421604)

9 Higham CE, Thomas JD, Bidlingmaier M, Drake WM \& Trainer PJ. Successful use of weekly pegvisomant administration in patients with acromegaly. European Journal of Endocrinology 2009161 21-25. (doi:10.1530/EJE-08-0990)

10 Elmlinger MW, Kuhnel W, Weber MM \& Ranke MB. Reference ranges for two automated chemiluminescent assays for serum insulin-like growth factor I (IGF-I) and IGF-binding protein 3 (IGFBP-3). Clinical Chemistry and Laboratory Medicine 200442 654-664.

11 Jorgensen JO, Feldt-Rasmussen U, Frystyk J, Chen JW, Kristensen LO, Hagen C \& Orskov H. Cotreatment of acromegaly with a somatostatin analog and a growth hormone receptor antagonist. Journal of Clinical Endocrinology and Metabolism 200590 5627-5631.

12 Freda PU, Gordon MB, Kelepouris N, Jonsson P, Koltowska-Haggstrom $\mathrm{M}$ \& van der Lely AJ. Long-term treatment with pegvisomant as monotherapy in patients with acromegaly: experience from acrostudy. Endocrine Practice 201521 264-274. (doi:10.4158/EP14330.OR)

13 Antonijoan RM, Barbanoj MJ, Cordero JA, Peraire C, Obach R, Valles J, Cherif-Cheikh R, Torres ML, Bismuth F \& Montes M. Pharmacokinetics of a new Autogel formulation of the somatostatin analogue lanreotide after a single subcutaneous dose in healthy volunteers. Journal of Pharmaceutics \& Pharmacology 200456 471-476. (doi:10.1211/0022357023123)

14 Bronstein M, Musolino N, Jallad R, Cendros JM, Ramis J, Obach R, Leselbaum A \& Catus F. Pharmacokinetic profile of lanreotide Autogel in patients with acromegaly after four deep subcutaneous injections of 60, 90 or 120 mg every 28 days. Clinical Endocrinology 200563 514-519. (doi:10.1111/cen.2005.63.issue-5)

15 Muto C, Chiba K \& Suwa T. Population pharmacokinetic and pharmacodynamic modeling of pegvisomant in asian and Western acromegaly patients. Journal of Clinical Pharmacology 201151 1628-1643. (doi:10.5414/CP201614)

Received 24 November 2015

Revised version received 9 February 2016

Accepted 19 February 2016 
Downloaded from Bioscientifica.com at $04 / 26 / 2023$ 11:55:28AM

via free access 